\title{
Adversarial Attacks and Defences Competition
}

Alexey Kurakin and Ian Goodfellow and Samy Bengio and Yinpeng Dong and Fangzhou Liao and Ming Liang and Tianyu Pang and Jun Zhu and Xiaolin $\mathrm{Hu}$ and Cihang Xie and Jianyu Wang and Zhishuai Zhang and Zhou Ren and Alan Yuille and Sangxia Huang and Yao Zhao and Yuzhe Zhao and Zhonglin Han and Junjiajia Long and Yerkebulan Berdibekov and Takuya Akiba and Seiya Tokui and Motoki Abe

\begin{abstract}
To accelerate research on adversarial examples and robustness of machine learning classifiers, Google Brain organized a NIPS 2017 competition that encouraged researchers to develop new methods to generate adversarial examples as well as to develop new ways to defend against them. In this chapter, we describe the
\end{abstract}

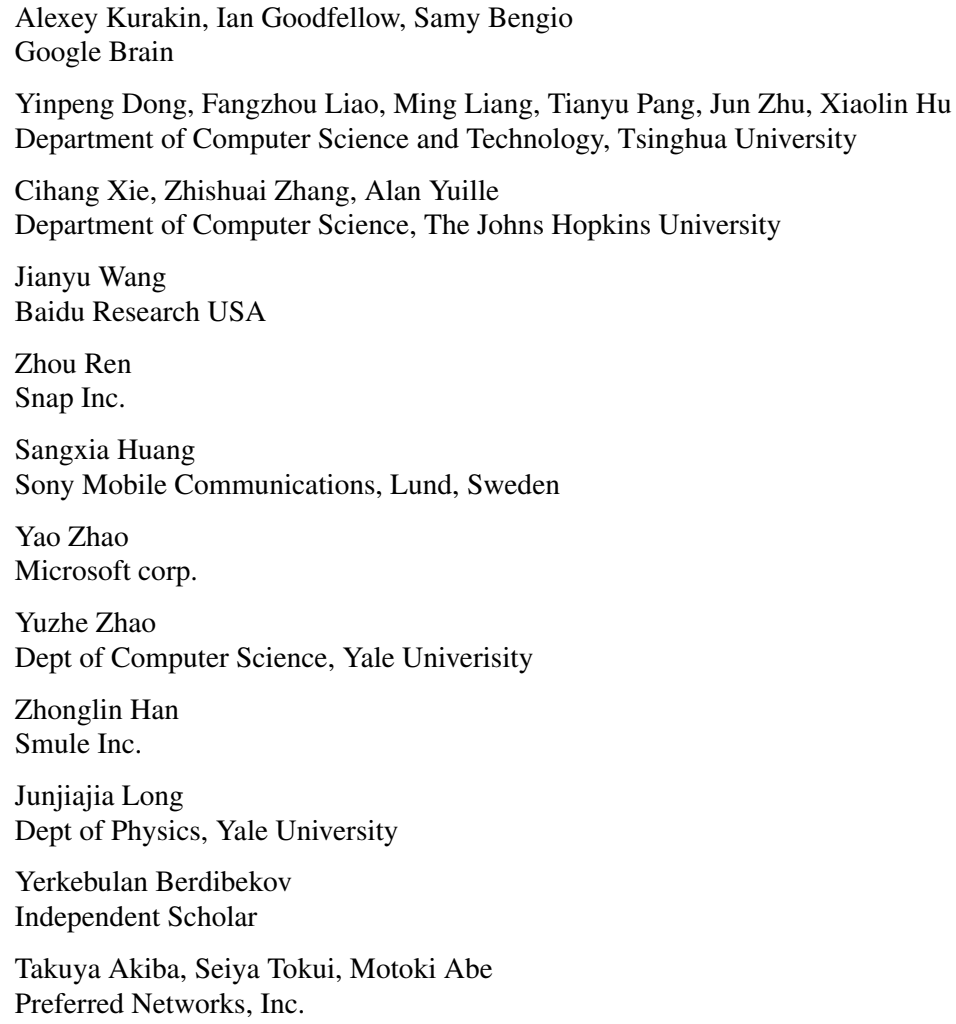


structure and organization of the competition and the solutions developed by several of the top-placing teams.

\section{Introduction}

Recent advances in machine learning and deep neural networks enabled researchers to solve multiple important practical problems like image, video, text classification and others.

However most existing machine learning classifiers are highly vulnerable to adversarial examples [2, 39, 15, 29]. An adversarial example is a sample of input data which has been modified very slightly in a way that is intended to cause a machine learning classifier to misclassify it. In many cases, these modifications can be so subtle that a human observer does not even notice the modification at all, yet the classifier still makes a mistake.

Adversarial examples pose security concerns because they could be used to perform an attack on machine learning systems, even if the adversary has no access to the underlying model.

Moreover it was discovered [22, 33] that it is possible to perform adversarial attacks even on a machine learning system which operates in physical world and perceives input through inaccurate sensors, instead of reading precise digital data.

In the long run, machine learning and AI systems will become more powerful. Machine learning security vulnerabilities similar to adversarial examples could be used to compromise and control highly powerful AIs. Thus, robustness to adversarial examples is an important part of the AI safety problem.

Research on adversarial attacks and defenses is difficult for many reasons. One reason is that evaluation of proposed attacks or proposed defenses is not straightforward. Traditional machine learning, with an assumption of a training set and test set that have been drawn i.i.d., is straightforward to evaluate by measuring the loss on the test set. For adversarial machine learning, defenders must contend with an openended problem, in which an attacker will send inputs from an unknown distribution. It is not sufficient to benchmark a defense against a single attack or even a suite of attacks prepared ahead of time by the researcher proposing the defense. Even if the defense performs well in such an experiment, it may be defeated by a new attack that works in a way the defender did not anticipate. Ideally, a defense would be provably sound, but machine learning in general and deep neural networks in particular are difficult to analyze theoretically. A competition therefore gives a useful intermediate form of evaluation: a defense is pitted against attacks built by independent teams, with both the defense team and the attack team incentivized to win. While such an evaluation is not as conclusive as a theoretical proof, it is a much better simulation of a real-life security scenario than an evaluation of a defense carried out by the proposer of the defense.

In this report, we describe the NIPS 2017 competition on adversarial attack and defense, including an overview of the key research problems involving adversarial 
examples (section 2), the structure and organization of the competition (section 3), and several of the methods developed by the top-placing competitors (section 4 ).

\section{Adversarial examples}

Adversarial examples are inputs to machine learning models that have been intentionally optimized to cause the model to make a mistake. We call an input example a "clean example" if it is a naturally occurring example, such as a photograph from the ImageNet dataset. If an adversary has modified an example with the intention of causing it to be misclassified, we call it an "adversarial example." Of course, the adversary may not necessarily succeed; a model may still classify the adversarial example correctly. We can measure the accuracy or the error rate of different models on a particular set of adversarial examples.

\subsection{Common attack scenarios}

Scenarios of possible adversarial attacks can be categorized along different dimensions.

First of all, attacks can be classified by the type of outcome the adversary desires:

- Non-targeted attack. In this the case adversary's goal is to cause the classifier to predict any inccorect label. The specific incorrect label does not matter.

- Targeted attack. In this case the adversary aims to change the classifier's prediction to some specific target class.

Second, attack scenarios can be classified by the amount of knowledge the adversary has about the model:

- White box. In the white box scenario, the adversary has full knowledge of the model including model type, model architecture and values of all parameters and trainable weights.

- Black box with probing. In this scenario, the adversary does not know very much about the model, but can probe or query the model, i.e. feed some inputs and observe outputs. There are many variants of this scenario-the adversary may know the architecture but not the parameters or the adversary may not even know the architecture, the adversary may be able to observe output probabilities for each class or the adversary may only be to observe the choice of the most likely class.

- Black box without probing In the black box without probing scenario, the adversary has limited or no knowledge about the model under attack and is not allowed to probe or query the model while constructing adversarial examples. In this case, the attacker must construct adversarial examples that fool most machine learning models. 
Third, attacks can be classifier by the way adversary can feed data into the model:

- Digital attack. In this case, the adversary has direct access to the actual data fed into the model. In other words, the adversary can choose specific float 32 values as input for the model. In a real world setting, this might occur when an attacker uploads a PNG file to a web service, and intentionally designs the file to be read incorrectly. For example, spam content might be posted on social media, using adversarial perturbations of the image file to evade the spam detector.

- Physical attack. In the case of an attack in the physical world, the adversary does not have direct access to the digital representation of provided to the model. Instead, the model is fed input obtained by sensors such as a camera or microphone. The adversary is able to place objects in the physical environment seen by the camera or produce sounds heard by the microphone. The exact digital representation obtained by the sensors will change based on factors like the camera angle, the distance to the microphone, ambient light or sound in the environment, etc. This means the attacker has less precise control over the input provided to the machine learning model.

\subsection{Attack methods}

Most of the attacks discussed in the literature are geared toward the white-box digital case.

\subsubsection{White box digital attacks}

L-BFGS . One of the first methods to find adversarial examples for neural networks was proposed in [39]. The idea of this method is to solve the following optimization problem:

$$
\left|x^{a d v}-x\right|_{2} \rightarrow \text { minimum, } \quad \text { s.t. } \quad f\left(x^{a d v}\right)=y_{\text {target }}, \quad x^{a d v} \in[0,1]^{m}
$$

The authors proposed to use the L-BFGS optimization method to solve this problem, thus the name of the attack.

One of the main drawbacks of this method is that it is quite slow. The method is not designed to counteract defenses such as reducing the number of bits used to store each pixel. Instead, the method is designed to find the smallest possible attack perturbation. This means the method can sometimes be defeated merely by degrading the image quality, for example, by rounding to an 8-bit representation of each pixel.

Fast gradient sign method (FGSM). To test the idea that adversarial examples can be found using only a linear approximation of the target model, the authors of [15] introduced the fast gradient sign method (FGSM). 
FGSM works by linearizing loss function in $L_{\infty}$ neighbourhood of a clean image and finds exact maximum of linearized function using following closed-form equation:

$$
x^{a d v}=x+\varepsilon \operatorname{sign}\left(\nabla_{x} J\left(x, y_{\text {true }}\right)\right)
$$

Iterative attacks The L-BFGS attack has a high success rate and high computational cost. The FGSM attack has a low success rate (especially when the defender anticipates it) and low computational cost. A nice tradeoff can be achieved by running iterative optimization algorithms that are specialized to reach a solution quickly, after a small number (e.g. 40) of iterations.

One strategy for designing optimization algorithms quickly is to take the FGSM (which can often reach an acceptable solution in one very large step) and run it for several steps but with a smaller step size. Because each FGSM step is designed to go all the way to the edge of a small norm ball surrounding the starting point for the step, the method makes rapid progress even when gradients are small. This leads to the Basic Iterative Method (BIM) method introduced in [23], also sometimes called Iterative FGSM (I-FGSM):

$$
x_{0}^{a d v}=\boldsymbol{X}, \quad x_{N+1}^{a d v}=\operatorname{Clip}_{X, \varepsilon}\left\{\boldsymbol{X}_{N}^{a d v}+\alpha \operatorname{sign}\left(\nabla_{X} J\left(\boldsymbol{X}_{N}^{a d v}, y_{t r u e}\right)\right)\right\}
$$

The BIM can be easily made into a target attack, called the Iterative Target Class Method:

$$
\boldsymbol{X}_{0}^{a d v}=\boldsymbol{X}, \quad \boldsymbol{X}_{N+1}^{a d v}=\operatorname{Clip}_{X, \varepsilon}\left\{\boldsymbol{X}_{N}^{a d v}-\alpha \operatorname{sign}\left(\nabla_{X} J\left(\boldsymbol{X}_{N}^{a d v}, y_{\text {target }}\right)\right)\right\}
$$

It was observed that with sufficient number of iterations this attack almost always succeeds in hitting target class [23].

Madry et. al's Attack [27] showed that the BIM can be significantly improved by starting from a random point within the $\varepsilon$ norm ball. This attack is often called projected gradient descent, but this name is somewhat confusing because (1) the term "projected gradient descent" already refers to an optimization method more general than the specific use for adversarial attack, (2) the other attacks use the gradient and perform project in the same way (the attack is the same as BIM except for the starting point) so the name doesn't differentiate this attack from the others.

Carlini and Wagner attack $(\mathbf{C} \& W)$. N. Carlini and D. Wagner followed a path of L-BFGS attack. They designed a loss function which has smaller values on adversarial examples and higher on clean examples and searched for adversarial examples by minimizing it [6]. But unlike [39] they used Adam [21] to solve the optimization problem and dealt with box constraints either by change of variables (i.e. $x=0.5(\tanh (w)+1)$ ) or by projecting results onto box constraints after each step.

They explored several possible loss functions and achieved the strongest $L_{2}$ attack with following: 


$$
\left\|x^{a d v}-x\right\|_{p}+c \max \left(\max _{i \neq Y} f\left(x^{a d v}\right)_{i}-f\left(x^{a d v}\right)_{Y},-\kappa\right) \rightarrow \text { minimum }
$$

where $x^{a d v}$ parametrized $0.5(\tanh (w)+1) ; Y$ is a shorter notation for target class $y_{\text {target }} ; c$ and $\kappa$ are method parameters.

Adversarial transformation networks (ATN). Another approach which was explored in [1] is to train a generative model to craft adversarial examples. This model takes a clean image as input and generates a corresponding adversarial image. One advantage of this approach is that, if the generative model itself is designed to be small, the ATN can generate adversarial examples faster than an explicit optimization algorithm. In theory, this approach can be faster than even the FGSM, if the ATN is designed to use less computation is needed for running back-propagation on the target model. (The ATN does of course require extra time to train, but once this cost has been paid an unlimited number of examples may be generated at low cost)

Attacks on non differentiable systems. All attacks mentioned about need to compute gradients of the model under attack in order to craft adversarial examples. However this may not be always possible, for example if model contains nondifferentiable operations. In such cases, the adversary can train a substitute model and utilize transferability of adversarial examples to perform an attack on nondifferentiable system, similar to black box attacks, which are described below.

\subsubsection{Black box attacks}

It was observed that adversarial examples generalize between different models [38]. In other words, a significant fraction of adversarial examples which fool one model are able to fool a different model. This property is called "transferability" and is used to craft adversarial examples in the black box scenario. The actual number of transferable adversarial examples could vary from a few percent to almost $100 \%$ depending on the source model, target model, dataset and other factors. Attackers in the black box scenario can train their own model on the same dataset as the target model, or even train their model on another dataset drawn from the same distribution. Adversarial examples for the adversary's model then have a good chance of fooling an unknown target model.

It is also possible to intentionally design models to systematically cause high transfer rates, rather than relying on luck to achieve transfer.

If the attacker is not in the complete black box scenario but is allowed to use probes, the probes may be used to train the attacker's own copy of the target model [30, 29] called a "substitute." This approach is powerful because the input examples sent as probes do not need to be actual training examples; instead they can be input points chosen by the attacker to find out exactly where the target model's decision boundary lies. The attacker's model is thus trained not just to be a good classifier but to actually reverse engineer the details of the target model, so the two models are systematically driven to have a high amount of transfer. 
In the complete black box scenario where the attacker cannot send probes, one strategy to increase the rate of transfer is to use an ensemble of several models as the source model for the adversarial examples [26]. The basic idea is that if an adversarial example fools every model in the ensemble, it is more likely to generalize and fool additional models.

Finally, in the black box scenario with probes, it is possible to just run optimization algorithms that do not use the gradient to directly attack the target model [3, 7]. The time required to generate a single adversarial example is generally much higher than when using a substitute, but if only a small number of adversarial examples are required, these methods may have an advantage because they do not have the high initial fixed cost of training the substitute.

\subsection{Overview of defenses}

No method of defending against adversarial examples is yet completely satisfactory. This remains a rapidly evolving research area. We given an overview of the (not yet fully succesful defense methods) proposed so far.

Since adversarial perturbations generated by many methods look like highfrequency noise to a human observer ${ }^{1}$ multiple authors have suggested to use image preprocessing and denoising as a potential defence against adversarial examples. There is a large variation in the proposed preprocessing techniques, like doing JPEG compression [9] or applying median filtering and reducing precision of input data [43]. While such defences may work well against certain attacks, defenses in this category have been shown to fail in the white box case, where the attacker is aware of the defense [19]. In the black box case, this defense can be effective in practice, as demonstrated by the winning team of the defense competition. Their defense, described in section 5.1, is an example of this family of denoising strategies.

Many defenses, intentionally or unintentionally, fall into a category called "gradient masking." Most white box attacks operate by computing gradients of the model and thus fail if it is impossible to compute useful gradients. Gradient masking consists of making the gradient useless, either by changing the model in some way that makes it non-differentiable or makes it have zero gradients in most places, or make the gradients point away from the decision boundary. Essentially, gradient masking means breaking the optimizer without actually moving the class decision boundaries substantially. Because the class decision boundaries are more or less the same, defenses based on gradient masking are highly vulnerable to black box transfer [30]. Some defense strategies (like replacing smooth sigmoid units with hard threshold units) are intentionally designed to perform gradient masking. Other defenses, like

\footnotetext{
${ }^{1}$ This may be because the human perceptual system finds the high-frequency components to be more salient; when blurred with a low pass filter, adversarial perturbations are often found to have significant low-frequency components
} 
many forms of adversarial training, are not designed with gradient masking as a goal, but seem to often learn to do gradient masking when applied in practice.

Many defenses are based on detecting adversarial examples and refusing to classify the input if there are signs of tampering [28]. This approach works long as the attacker is unaware of the detector or the attack is not strong enough. Otherwise the attacker can construct an attack which simultaneously fools the detector into thinking an adversarial input is a legitimate input and fools the classifier into making the wrong classification [5].

Some defenses work but do so at the cost of seriously reducing accuracy on clean examples. For example, shallow RBF networks are highly robust to adversarial examples on small datasets like MNIST [16] but have much worse accuracy on clean MNIST than deep neural networks. Deep RBF networks might be both robust to adversarial examples and accurate on clean data, but to our knowledge no one has successfully trained one.

Capsule networks have shown robustness to white box attacks on the SmallNORB dataset, but have not yet been evaluated on other datasets more commonly used in the adversarial example literature [13].

The most popular defense in current research papers is probably adversarial training [38, 15, 20]. The idea is to inject adversarial examples into training process and train the model either on adversarial examples or on mix of clean and adversarial examples. The approach was successfully applied to large datasets [24], and can be made more effective by using discrete vector code representations rather than real number representations of the input [4]. One key drawback of adversarial training is that it tends to overfit to the specific attack used at training time. This has been overcome, at least on small datasets, by adding noise prior to starting the optimizer for the attack [27]. Another key drawback of adversarial training is that it tends to inadvertently learn to do gradient masking rather than to actually move the decision boundary. This can be largely overcome by training on adversarial examples drawn from an ensemble of several models [40]. A remaining key drawback of adversarial training is that it tends to overfit to specific constraint region used to generate the adversarial examples (models trained to resist adversarial examples in a max-norm ball may not resist adversarial examples based on large modifications to background pixels [14] even if the new adversarial examples do not appear particularly challenging to a human observer).

\section{Adversarial competition}

The phenomenon of adversarial examples creates a new set of problems in machine learning. Studying these problems is often difficult, because when a researcher proposes a new attack, it is hard to tell whether their attack is strong, or whether they have not implemented their defense method used for benchmarking well enough. Similarly, it is hard to tell whether a new defense method works well or whether it has just not been tested against the right attack. 
To accelerate research in adversarial machine learning and pit many proposed attacks and defenses against each other in order to obtain the most vigorous evaluation possible of these methods, we decided to organize a competition.

In this competition participants are invited to submit methods which craft adversarial examples (attacks) as well as classifiers which are robust to adversarial eaxmples (defenses). When evaluating competition, we run all attack methods on our dataset to produce adversarial examples and then run all defenses on all generated adversarial examples. Attacks are ranked by number of times there were able to fool defenses and defenses are scored by number of correctly classified examples.

\subsection{Dataset}

When making a dataset for these competition we had following requirements:

1. Large enough dataset and non-trivial problem, so the competition would be interesting.

2. Well known problem, so people potentially can reuse existing classifiers. (This ensures that competitors are able to focus on the adversarial nature of the challenge, rather than spending all their time coming up with a solution to the underlying task)

3. Data samples which were never used before, so participants unlikely to overfit to well known dataset.

These requirements were satisfied by choosing image classification problem and creating a dataset with ImageNet-compatible images [10]. To do this we collected a set of images which were never used in publicly available datasets, labelled them using pretrained ImageNet classifier and then manually verified that these labels are correct.

The original (non-adversarial) ImageNet challenge [32] is a complex and interesting problem, and thus satisfies requirement number 1 . Additionally there are plenty of existing classifiers for ImageNet data, which satisfies requirement number 2. Because we collected new images and labelled them instead of using the already available dataset, our approach satisfies requirement number 3 .

Overall we collected two datasets for this competitions:

- DEV dataset was released to participants in the beginning of the competition, so they can use it for development of their solutions. This dataset contained 1000 images.

- FINAL dataset was kept secret and was used to evaluate final submissions of participants. It contained 5000 images. 


\subsection{Tasks and competition rules}

Our competition had three tracks. Each track had a different task:

- Non-targeted adversarial attack. In this track participants were invited to submit a method which performs a non-targeted black box attack, i.e. given an input image, generate an adversarial image which is likely be misclassified by unknown classifier.

- Targeted adversarial attack. In this track participants were invited to submit a method which performs a targeted black box attack, i.e. given an input image and a target class, generate an adversarial image which is likely be misclassified as a given target class by unknown classifier.

- Defense against adversarial attacks. In this track, participants were invited to submit a classifier which is robust to adversarial examples.

In all tracks participants submitted a program which performs the given task and we ran this code using our evaluation infrastructure.

The submitted program was given a set of images (and target classes in case of targeted attack) as an input, and had to produce either an adversarial image (for attack submission) or classification label (for defense submission) for each input image.

Input images were fed to each submission in batches of 100 images. To limit the budget required for computation, the rules of the competitions specified that each submission had to process a batch of 100 images in no more than 500 seconds. If a submission was not done after 500 seconds, then processing of the current batch was interrupted and the submission was fed the next batch. Only successfully processed images were included in the score of the submission (see details below in evaluation sub-section).

\subsection{Evaluation metrics}

An attack got 1 point each time it was able to fool a defense on single image. If attack was unable to fool a defense or if it failed to generate an adversarial version of an image within the time limit then it got 0 points for that image. A defense got 1 point for each correctly classified image and 0 points for incorrect classification or failure to produce a classification label. A defense was used to score attacks only if it successfully processed all input images, so that attackers were neither rewarded nor penalized for making images that caused the defender to crash or time out- the competition is purely about classification accuracy. Likewise, an attack was used to score defenses only if it succesfully processed all inputs. Points for each submission were added together and then normalized (using the same normalization constant for all submissions), such that final scores of all submissions are in the range $[0,1]$, where 1 means success on all images and 0 means failure on all images. 
Specifically, the score of each non-targeted attack a(Image) was computed using the following formula:

$$
\frac{1}{|D| N} \sum_{d \in D} \sum_{k=1}^{N}\left[d\left(a\left(I_{k}\right)\right) \neq \text { TrueLabel }_{k}\right]
$$

The score of each targeted attack a (Image, TargetLabel) was computed as:

$$
\frac{1}{|D| N} \sum_{d \in D} \sum_{k=1}^{N}\left[d\left(a\left(I_{k}, \text { Target }_{\text {Label }}\right)\right)=\text { Target }_{\text {Label }}\right]
$$

The score of each defense $d$ (Image) was computed in the following way:

$$
\frac{1}{|A| N} \sum_{a \in A} \sum_{k=1}^{N}\left[d\left(a\left(I_{k}\right)\right)=\text { TrueLabel }_{k}\right]
$$

Where:

- $A$ is the set of all attacks (targeted and non-targeted) which were used for evaluation of defenses (attacks that crashed on some inputs or ran out of time for some inputs were not used to evaluate defense);

- $D$ is the set of all defenses which were used for evaluation of attacks (defenses that crashed on some inputs or ran out of time for some inputs were not used to evaluate attacks);

- $N$ is the number of images in the dataset;

- $I_{k}$ is the $k$-th image from the dataset;

- TrueLabelk is the true label of image $I_{k}$;

- Target Label $k_{k}$ is the chosen target label for image $k$;

- $[P]$ is an indicator function which equals to 1 when $P$ is true, and 0 when $P$ is false or undefined.

- $d(\bullet)$ is a defending classifier. If the binary fails to complete execution within the time limit, the output of $d(\bullet)$ is a null label that never equals the true label. If $d(\bullet)$ is called on an undefined image, it is defined to always return the true label, so an attacker that crashes receives zero points.

Additionally to metrics used for ranking, after the competition we computed worst case score for each submission in defense and non-targeted attack tracks. These scores were useful to understand how submissions act in the worst case. To compute worst score of defense we computed accuracy of the defense against each attack and chosen minimum:

$$
\frac{1}{N} \min _{a \in A} \sum_{k=1}^{N}\left[d\left(a\left(I_{k}\right)\right)=\text { TrueLabel }_{k}\right]
$$

To compute worst case score of non-targeted attack we computed how often attack caused misclassification when used against each defense and chosen minimum misclassification rate: 


$$
\frac{1}{N} \min _{d \in D} \sum_{k=1}^{N}\left[d\left(a\left(I_{k}\right)\right) \neq \text { TrueLabel }_{k}\right]
$$

Worst case score of targeted attack could be computed in a similar way, but generally not useful because targeted attacks are much weaker than non-targeted and all worst scores of targeted attacks were 0 .

\subsection{Competition schedule}

The competition was announced in May 2017, launched in the beginning of July 2017 and finished on October 1st, 2017. The ompetition was run in multiple rounds. There were three development rounds followed by the final round:

- August 1, 2017 - first development round

- September 1, 2017 - second development round

- September 15, 2017 - third development round

- October 1, 2017 - deadline for final submission

Development rounds were optional and their main purpose was to help participants to test their solution. Only the final round was used to compute final scores of submissions and determine winners.

All rounds were evaluated in a similar way. For the evaluation of the round we gathered all submissions which were submitted before the round deadline, ran all of them and computed scores as described in section 3.3

We used DEV dataset to compute scores in development rounds and secret FINAL dataset to compute scores in the final round.

\subsection{Technical aspects of evaluation}

Competition participants were submitting pieces of code and we have run them ourselves. This approach posess several challanges. First of all we need to protect competition infrastructure from malicious code. Secondly, given the dataset size and number of submissions we had to run it in an efficient way.

We partnered with Kaggle ${ }^{2}$ and used their platform as a frontend for the competition. Kaggle hosted competition web-site, leaderboard and participants were uploading submissions through Kaggle. For evaluation of each round we took all submissions from Kaggle and fed them into our evaluation infrastructure.

Evaluation infrastructure worked as described in algorithm 1 . As could be seen from the algorithm, attacks could be run independently of each other. Same for defenses. We took advantage of this fact and parallelized execution of all attacks and all defenses by spreading them across multiple machines.

2 WwW.kaggle.com 


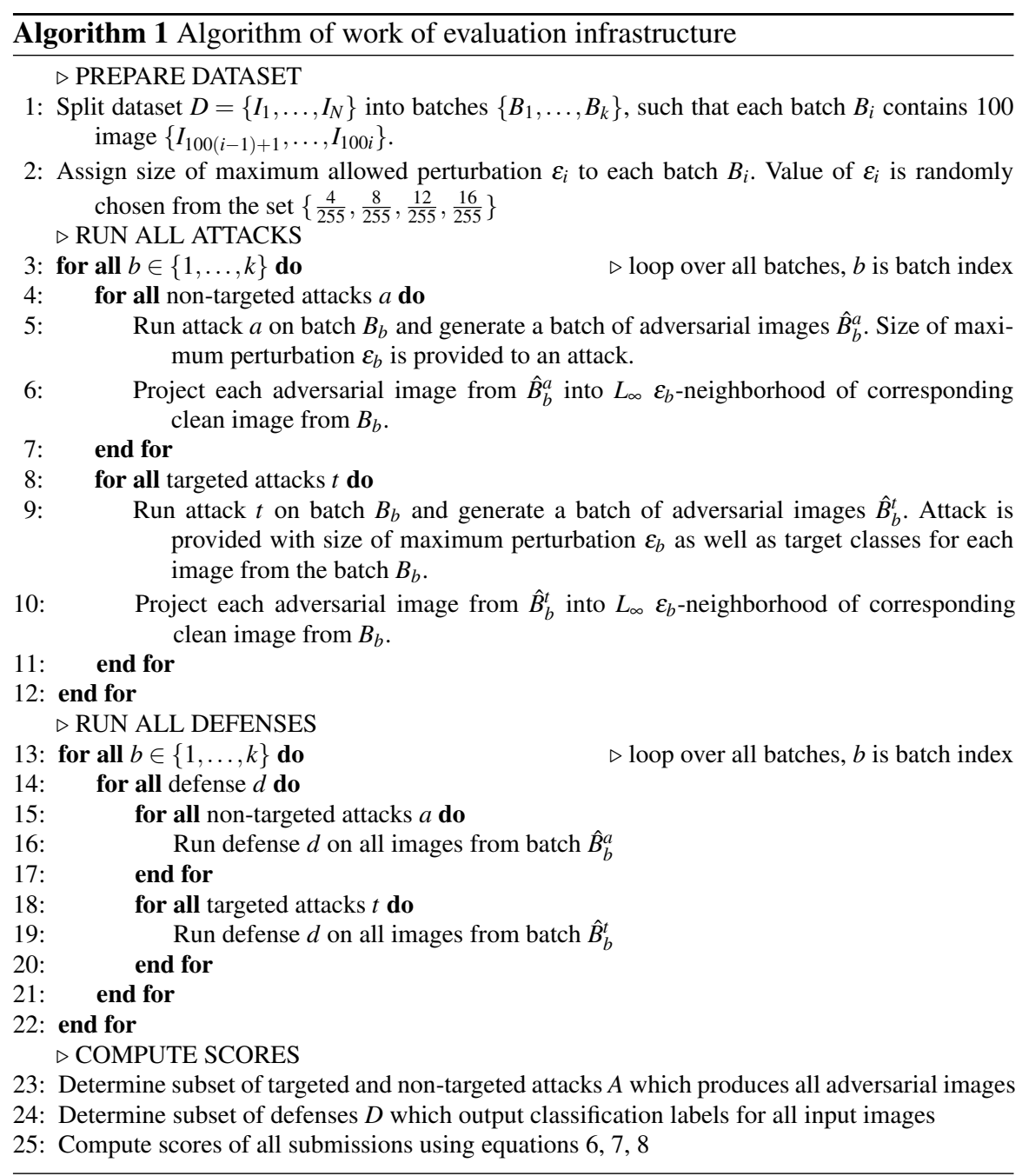

For final evaluation we used 100 Google Cloud VMs. At any given moment one VM was running either one attack on one batch from the dataset or one defense on one batch of adversarial images. Submissions were run inside Docker containers to isolate submissions from our evaluation infrastructure and from the outside world.

\section{Competition results}

For the final round we had 91 non-targeted attack submissions, 65 targeted attack submission and 107 defense submissions. During the course of competitions sub- 


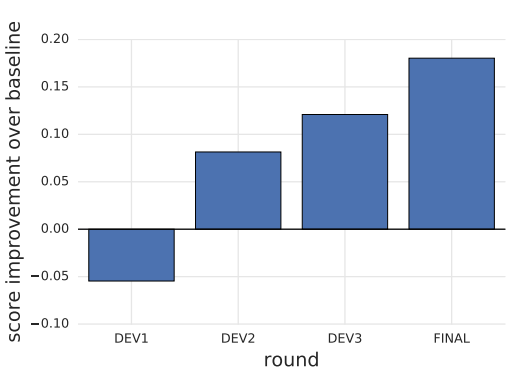

(a) Defenses

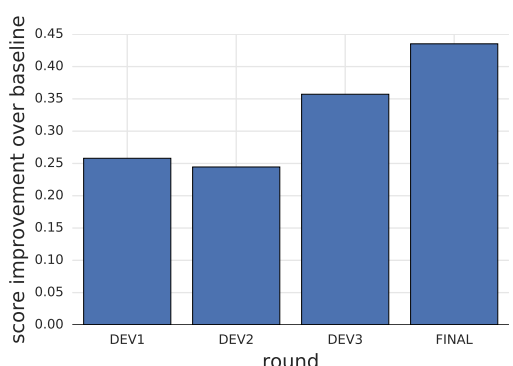

(b) Non-targeted attacks

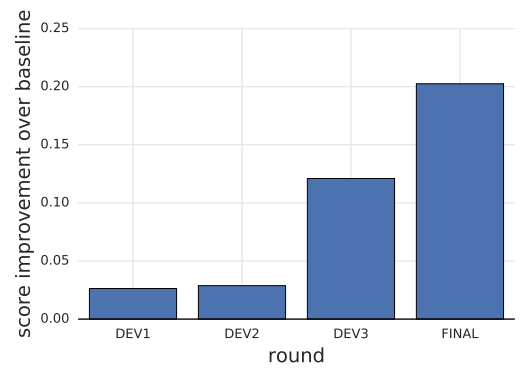

(c) Targeted attacks

Fig. 1: Plots which shows difference between score of top submission and best baseline in each round in each track. As could be seen from the plot, submissions kept improving each round.

missions kept improving their results compared to baselines which could be seen from Figure 1 .

Final results of the top submissions in each track are provided in tables 1,2 and 3 . Meaning of the columns is following. Rank is submission rank in final scoring, score is submission score as described in Section 3.3, raw score is un-normalized score which is number of times submission got a point on the image, worst score is submission score in the worst case and medial eval time is median time needed for evaluation of one batch of 100 images. To put things into prospective, plots of all submission scores in final round from best to worst and comparison with provided baselines are depicted in Figure 2

As could be seen from the tables, best defenses achieved more than $90 \%$ accuracy on all adversarial images from all attacks. At the same time worst case scores of defenses are much lower. The highest worst case score among all defenses is only $53.6 \%$.

This could be an indication that it's possible to achieve pretty high accuracy against adversarial examples in average case, however model will still be suspectible to adversarial examples and could be fooled if adversary will be able to find them. 
Table 1: Top-5 defense submissions, best baseline and submission with maximum worst-case score

\begin{tabular}{|c|c|c|c|c|}
\hline Rank|Team name or baseline & Score & aw Score & |Worst Score| & val time \\
\hline 1|TSAIL & |0.953164| & 691044 & 0.1184 & 51.0 \\
\hline 2 iyswim & 0.923524 & 669555 & 0.2520 & 126.0 \\
\hline 3 Anil Thomas & 0.914840 & 663259 & 0.2666 & 97.0 \\
\hline 4 erko & 0.911961 & 661172 & 0.2920 & 87.0 \\
\hline \begin{tabular}{l|l}
5 & Stanford \& Suns
\end{tabular} & 0.910593 & 660180 & 0.0682 & 129.0 \\
\hline 24 Rafael Moraes & 0.871739 & 632011 & 0.5358 & 17.0 \\
\hline 56 Baseline (Ens. adv. ir_v2) & 0.772908 & 560358 & 0.0186 & 17.0 \\
\hline
\end{tabular}

Table 2: Top-5 non-targeted attack submissions, best baseline and best submission with according to worst-case score.

\begin{tabular}{|c|c|c|c|c|}
\hline Rank|Team name or baseline & Score & aw Score & |Worst Score & Median eval time \\
\hline $1 \mid$ TSAIL & |0.781644 | & 410363 & 0.1364 & 423.0 \\
\hline 2 Sangxia & 0.776855 & 407849 & 0.3412 & 421.0 \\
\hline \begin{tabular}{l|l}
3 & Stanford \& Sun
\end{tabular} & 0.774025 & 406363 & 0.2722 & 497.0 \\
\hline 4 iwiwi & 0.768981 & 403715 & 0.1352 & 76.0 \\
\hline 5 toshi_k & 0.755598 & 396689 & 0.3322 & 448.0 \\
\hline 44 Baseline (FGSM) & $|0.346400|$ & 181860 & 0.0174 & 17.0 \\
\hline
\end{tabular}

Table 3: Top-5 targeted attack submissions and best baseline.

\begin{tabular}{|c|c|c|c|c|}
\hline \multicolumn{2}{|c|}{ Rank|Team } & \multicolumn{3}{|c|}{ Score $\mid$ Raw Score $\mid$ Median Eval Time } \\
\hline & |TSAIL & $|0.402211|$ & 211161.0 & 392.0 \\
\hline 2 & Sangxia & 0.368773 & 193606.0 & 414.0 \\
\hline 3 & FatFingers & 0.368029 & 193215.0 & 493.0 \\
\hline 4 & Anil Thomas & 0.364552 & 191390.0 & 495.0 \\
\hline 5 & WNP & 0.347935 & 182666.0 & 487.0 \\
\hline 24 & $\mid$ Baseline (Iter. T. C. 20) $\mid$ & $|0.199773|$ & 104881.0 & 127.0 \\
\hline
\end{tabular}

Similar observation holds for attacks. Best attacks achieved up to $78 \%$ fooling rate against all defenses, at the same time worst case score of attacks was no more than $34 \%$.

\section{Top scoring submissions}

In the remainder of the chapter, we present the solutions of several of the top-scoring teams.

To describe the solutions, we use the following notation: 


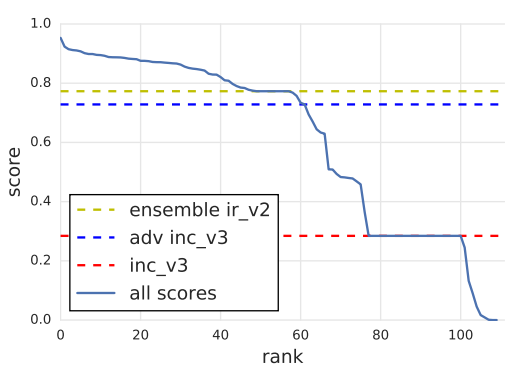

(a) Defenses

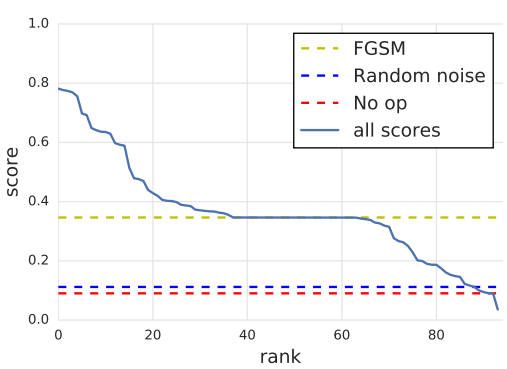

(b) Non-targeted attacks

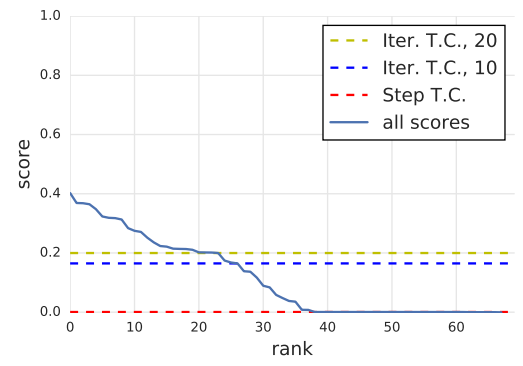

(c) Targeted attacks

Fig. 2: Plots with scores of submissions in all three tracks. Solid line of each plot is scores of submissions depending on submission rank. Dashed lines are scores of baselines we provided. These plots demonstrate difference between best and worst submissions as well as how much top submissions were able to improve provided baselines.

- $x$ - input image with label $y_{\text {true }}$. Different images are distinguished by superscripts, for examples images $x^{1}, x^{2}, \ldots$ with labels $y_{\text {true }}^{1}, y_{\text {true }}^{2}, \ldots$.

- $y_{\text {target }}$ is a target class for image $x$ for targeted adversarial attack.

- Functions with names like $f(\bullet), g(\bullet), h(\bullet), \ldots$ are classifiers which map input images into logits. In other words $f(x)$ is logits vector of networks $f$ on image $x$

- $J(f(x), y)$ - cross entropy loss between logits $f(x)$ and class $y$.

- $\varepsilon$ - maximum $L_{\infty}$ norm of adversarial perturbation.

- $x_{a d v}$-adversarial images. For iterative methods $x_{a d v}^{i}$ is adversarial example generated on step $i$.

- $\operatorname{Clip}_{[a, b]}(\bullet)$ is a function which performs element-wise clipping of input tensor to interval $[a, b]$.

- $\mathscr{X}$ is the set of all training examples.

All values of images are normalized to be in $[0,1]$ interval. Values of $\varepsilon$ are also normalized to $[0,1]$ range, for examples $\varepsilon=\frac{16}{255}$ correspond to uint 8 value of epsilon equal to 16 . 


\subsection{1st place in defense track: team TsAIL}

Team members: Yinpeng Dong, Fangzhou Liao, Ming Liang, Tianyu Pang, Jun Zhu and Xiaolin Hu.

In this section, we introduce the high-level representation guided denoiser (HGD) method, which won the first place in the defense track. The idea is to train a neural network based denoiser to remove the adversarial perturbation.

\subsubsection{Dataset}

To prepare the training set for the denoiser, we first extracted $20 \mathrm{~K}$ images from the ImageNet training set (20 images per class). Then we used a bunch of adversarial attacks to distort these images and form a training set. Attacking methods included FGSM and I-FGSM and were applied to the many models and their ensembles to simulate weak and strong attacks.

\subsubsection{Denoising U-net}

Denoising autoencoder (DAE) [41] is a potential choice of the denoising network. But DAE has a bottleneck for the transmission of fine-scale information between the encoder and decoder. This bottleneck structure may not be capable of carrying the multi-scale information contained in the images. That's why we used a denoising U-net (DUNET).

Compared with DAE, the DUNET adds some lateral connections from encoder layers to their corresponding decoder layers of the same resolution. In this way, the network is learning to predict adversarial noise only, which is more relevant to denoising and easier than reconstructing the whole image [44]. The clean image can be readily obtained by subtracting the noise from the corrupted input:

$$
\begin{gathered}
d \hat{x}=D_{w}\left(x^{a d v}\right) . \\
\hat{x}=x^{a d v}-d \hat{x} .
\end{gathered}
$$

where $D_{w}$ is a denoiser network with parameters $w, d \hat{x}$ is predicted adversarial noise and $\hat{x}$ is reconstructured clean image.

\subsubsection{Loss function}

The vanilla denoiser uses the reconstructing distance as the loss function, but we found a better method. Given a target neural network, we extract its representation at $l$-th layer for $x$ and $\hat{x}$, and calculate the loss function as:

$$
L=\left\|f_{l}(\hat{x})-f_{l}(x)\right\|_{1} .
$$


The corresponding models are called HGD, because the supervised signal comes from certain high-level layers of the classifier and carries guidance information related to image classification.

We propose two HGDs with different choices of $l$. For the first HGD, we define $l=-2$ as the index of the topmost convolutional layer. This denoiser is called feature guided denoiser (FGD). For the second HGD, we use the logits layer. So it is called logits guided denoiser (LGD).

Another kind of HGD uses the classification loss of the target model as the denoising loss function, which is supervised learning as ground truth labels are needed. This model is called class label guided denoiser (CGD). In this case the loss function is optimized with respect to the parameters of the denoiser $w$, while the parameters of the guiding model are fixed.

Please refer to our full-length paper [25] for more information.

\subsection{1st place in both attack tracks: team TsAIL}

Team members: Yinpeng Dong, Fangzhou Liao, Ming Liang, Tianyu Pang, Jun Zhu and Xiaolin Hu.

In this section, we introduce the momentum iterative gradient-based attack method, which won the first places in both the non-targeted attack and targeted attack tracks. We first describe the algorithm in Sec. 5.2.1, and then illustrate our submissions for non-targeted and targeted attacks respectively in Sec. 5.2.2 and Sec. 5.2.3 A more detailed description can be found in [11].

\subsubsection{Method}

The momentum iterative attack method is built upon the basic iterative method [23], by adding a momentum term to greatly improve the transferability of the generated adversarial examples.

Existing attack methods exhibit low efficacy when attacking black-box models, due to the well-known trade-off between the attack strength and the transferability [24]. In particular, one-step method (e.g., FGSM) calculates the gradient only once using the assumption of linearity of the decision boundary around the data point. However in practice, the linear assumption may not hold when the distortions are large [26], which makes the adversarial examples generated by one-step method "underfit" the model, limiting attack strength. In contrast, basic iterative method greedily moves the adversarial example in the direction of the gradient in each iteration. Therefore, the adversarial example can easily drop into poor local optima and "overfit" the model, which are not likely to transfer across models.

In order to break such a dilemma, we integrate momentum [31] into the basic iterative method for the purpose of stabilizing update directions and escaping from poor local optima, which are the common benefits of momentum in optimization 
literature [12, 34]. As a consequence, it alleviates the trade-off between the attack strength and the transferability, demonstrating strong black-box attacks.

The momentum iterative method for non-targeted attack is summarized as:

$$
g^{t+1}=\mu \cdot g^{t}+\frac{\nabla_{x} J\left(f\left(x_{a d v}^{t}\right), y_{\text {true }}\right)}{\left\|\nabla_{x} J\left(f\left(x_{\text {adv }}^{t}\right), y_{\text {true }}\right)\right\|_{1}}, x_{a d v}^{t+1}=\operatorname{Clip}_{[0,1]}\left(x_{a d v}^{t}+\alpha \cdot \operatorname{sign}\left(g^{t+1}\right)\right)
$$

where $g^{0}=0, x_{a d v}^{0}=x, \alpha=\frac{\varepsilon}{T}$ with T being the number of iterations. $g^{t}$ gathers the gradients of the first $t$ iterations with a decay factor $\mu$ and adversarial example $x_{a d v}^{t}$ is perturbed in the direction of the sign of $g^{t}$ with the step size $\alpha$. In each iteration, the current gradient $\nabla_{x} J\left(f\left(x_{a d v}^{t}\right), y_{\text {true }}\right)$ is normalized to have unit $L_{1}$ norm (however other norms will work too), because we noticed that the scale of the gradients varies in magnitude between iterations.

To obtain more transferable adversarial examples, we apply the momentum iterative method to attack an ensemble of models. If an example remains adversarial for multiple models, it may capture an intrinsic direction that always fools these models and is more likely to transfer to other models at the same time [26], thus enabling powerful black-box attacks.

We propose to attack multiple models whose logit activations are fused together, because the logits capture the logarithm relationships between the probability predictions, an ensemble of models fused by logits aggregates the fine detailed outputs of all models, whose vulnerability can be easily discovered. Specifically, to attack an ensemble of $K$ models, we fuse the logits as

$$
f(x)=\sum_{k=1}^{K} w_{k} f_{k}(x)
$$

where $f_{k}(x)$ are the $k$-th model, $w_{k}$ is the ensemble weight with $w_{k} \geq 0$ and $\sum_{k=1}^{K} w_{k}=1$. Therefore we get a big ensemble model $f(x)$ and we can use the momentum iterative method to attack $f$.

\subsubsection{Submission for non-targeted attack}

In non-targeted attack, we implemented the momentum iterative method for attacking an ensemble of following models:

- Normally trained (i.e. without adversarial training) Inception v3 [37, Inception v4 [35], Inception Resnet v2 [35] and Resnet v2-101 [18] models.

- Adversarially trained Inception $v 3_{\mathrm{adv}}[24]$ model.

- Ensemble adversarially trained Inc-v $3_{\text {ens } 3}$, Inc-v $3_{\text {ens } 4}$ and IncRes-v $2_{\text {ens }}$ models from [40].

Ensemble weights (from Equation 15 were $\frac{0.25}{7.25}$ for Inception-v $3_{\text {adv }}$ and $\frac{1}{7.25}$ for all other models. The number of iterations was 10 and the decay factor $\mu$ was 1.0. 


\subsubsection{Submission for targeted attack}

For targeted attacks, we used a different formula of momentum iterative method:

$$
\begin{aligned}
g^{t+1} & =\mu \cdot g^{t}+\frac{\nabla_{x} J\left(f\left(x_{a d v}^{t}\right), y_{\text {target }}\right)}{\operatorname{std}\left(\nabla_{x} J\left(f\left(x_{\text {adv }}^{t}\right), y_{\text {target }}\right)\right.} \\
x_{a d v}^{t+1} & =\operatorname{Clip}_{[0,1]}\left(x_{a d v}^{t}-\alpha \cdot \operatorname{Clip}_{[-2,2]}\left(\operatorname{round}\left(g^{t+1}\right)\right)\right)
\end{aligned}
$$

where $\operatorname{std}(\bullet)$ is the standard deviation and $\operatorname{round}(\bullet)$ is rounding to nearest integer. Values of $\operatorname{Clip}_{[-2,2]}(\operatorname{round}(\bullet))$ are in set $\{-2,-1,0,1,2\}$ which enables larger search space compared to sign function.

No transferability of the generated adversarial examples was observed in the targeted attacks, so we implement our method for attacking several commonly used white-box models.

We built two versions of the attacks. If the size of perturbation $\varepsilon$ was smaller than $\frac{8}{255}$, we attacked ensemble of Inception v 3 and IncRes-v 2 ens with weights $\frac{1}{3}$ and $\frac{2}{3}$; otherwise we attacked an ensemble of Inception v3, Inception-v $3_{\mathrm{adv}}$, Inc-v $3_{\text {ens } 3}$, Inc$\mathrm{v} 3_{\text {ens } 4}$ and IncRes-v 2 ens with ensemble weights $\frac{4}{11}, \frac{1}{11}, \frac{1}{11}, \frac{1}{11}$ and $\frac{4}{11}$. The number of iterations were 40 and 20 respectively, and the decay factor $\mu$ was 1.0.

\subsection{2nd place in defense track: team iyswim}

Team members: Cihang Xie, Jianyu Wang, Zhishuai Zhang, Zhou Ren and Alan Yuille

In this submission, we propose to utilize randomization as a defense against adversarial examples. Specifically, we propose a randomization-based method, as shown in figure 3, which adds a random resizing layer and a random padding layer to the beginning of the classification networks. Our method enjoys the following advantages: (1) no additional training or fine-tuning; (2) very few additional computations; (3) compatible with other adversarial defense methods. By combining the proposed randomization method with an adversarially trained model, it ranked No.2 in the NIPS adversarial defense challenge.

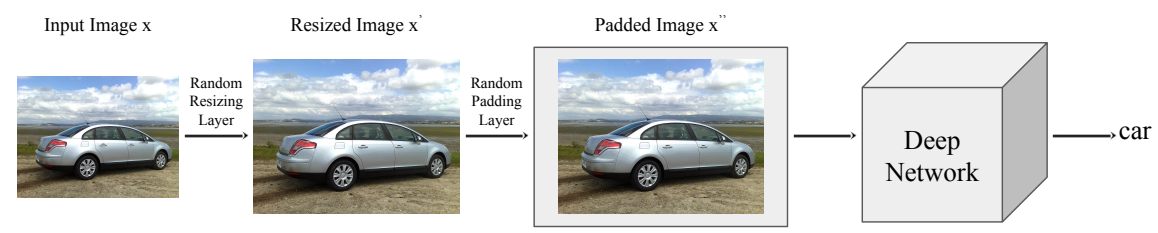

Fig. 3: The pipeline of the proposed defense method. The input image $x$ first goes through the random resizing layer with a random scale applied. Then the random padding layer pads the resized image $x^{\prime}$ in a random manner. The resulting padded image $x^{\prime \prime}$ is used for classification. 


\subsubsection{Randomization as defense}

Intuitively, the adversarial perturbation generated by iterative attacks may easily get over-fitted to the specific network parameters, and thus be less transferable. Due to this weak generalization ability, we hypothesis that low-level image transformations, e.g., resizing, padding, compression, etc, may probably destroy the specific structure of adversarial perturbations, thus making it a good defense. It can even defend against white-box iterative attacks if random transformations are applied. This is because each test image goes through a transformation randomly and the attacker does not know this specific transformation when generating adversarial noise.

\subsubsection{Randomization layers}

The first randomization layer is a random resizing layer, which resizes the original input image $x$ with the size $W \times H \times 3$ to a new image $x^{\prime}$ with random size $W^{\prime} \times H^{\prime} \times 3$. Note that, $\left|W^{\prime}-W\right|$ and $\left|H^{\prime}-H\right|$ should be within a reasonably small range, otherwise the network performance on clean images would significantly drop. Taking Inception-ResNet network [35] as an example, the original data input size is $299 \times 299 \times 3$. Empirically we found that the network accuracy hardly drops if we control the height and width of the resized image $x^{\prime}$ to be within the range $[299,331)$.

The second randomization layer is the random padding layer, which pads zeros around the resized image in a random manner. Specifically, by padding the resized image $x^{\prime}$ into a new image $x^{\prime \prime}$ with the size $W^{\prime \prime} \times H^{\prime \prime} \times 3$, we can choose to pad $w$ zero pixels on the left, $W^{\prime \prime}-W^{\prime}-w$ zero pixels on the right, $h$ zero pixels on the top and $H^{\prime \prime}-H^{\prime}-h$ zero pixels on the bottom. This results in a total number of $\left(W^{\prime \prime}-W^{\prime}+1\right) \times\left(H^{\prime \prime}-H^{\prime}+1\right)$ different possible padding patterns.

During implementation, the original image first goes through two randomization layers, and then we pass the transformed image to the original $\mathrm{CNN}$ for classification. The pipeline is illustrated in figure 3

\subsubsection{Randomization layers + adversarial training}

Recently, adversarial training [24, 40] was developed as an effective defense for single-step attacks. Thus by adding the proposed random transformations as additional layers to an adversarially trained model [40], it is expected that this method is able to effectively defend against both single-step and iterative attacks, including both black-box and white-box settings.

\subsubsection{Submission details and results}

An adversarially trained model appended with randomization layers was submitted as our defense model to the challenge. Specifically, we (1) set the resizing range to 
be $[310,331)$; (2) set the padding size to be $331 \times 331 \times 3$; (3) choose the adversarially trained model to be ens-adv-Inception-Resnet- $v 2^{3}$, (4) average the prediction results over 30 randomization patterns for each image; (5) flip the input image with probability 0.5 for each randomization pattern. The whole implementation is public available 4

By evaluating our model against 156 different attacks, it reaches a normalized score of 0.924 (ranked No.2 among 107 defense models), which is far better than using ensemble adversarial training [40] alone with a normalized score of 0.773 . This result further demonstrates that the proposed randomization method can effectively make deep networks much more robust to adversarial attacks.

\subsubsection{Attackers with more information}

When submitting the proposed defense method to the NIPS competition, the randomization layers are remained as an unknown network module for the attackers. We thus test the robustness of this defense method further by assuming that the attackers are aware of the existence of randomization layers. Extensive experiments are performed in [42], and it shows that the attackers still cannot break this defense completely in practice. Interested readers can refer to [42] for more details.

\subsection{2nd place in both attack tracks: team Sangxia}

\section{Team members: Sangxia Huang}

In this section, we present the submission by Sangxia Huang for both nontargeted and targeted attacks. The approach is an iterated FGSM attack against an ensemble of classifiers with random perturbations and augmentations for increased robustness and transferability of the generated attacks. The source code is available online. ${ }^{5}$ We also optimize the iteration steps for improved efficiency as we describe in more details below.

Basic idea An intriguing property of adversarial examples observed in many works [30, 38, 16, 29] is that adversarial examples generated for one classifier transfer to other classifiers. Therefore, a natural approach for effective attacks against unknown classifiers is to generate strong adversarial examples against a large collection of classifiers.

Let $f^{1}, \ldots, f^{k}$ be an ensemble of image classifiers that we choose to target. In our solution we give equal weights to each of them. For notation simplicity, we assume that the inputs to all $f^{i}$ have the same size. Otherwise, we first insert a bi-linear

\footnotetext{
3 https://download.tensorflow.org/models/ens_adv_inception_resnet_ v2_2017_08_18.tar.gz

4 https://github.com/cihangxie/NIPS2017_adv_challenge_defense

5 https://github.com/sangxia/nips-2017-adversarial
} 
scaling layer, which is differentiable. The differentiability ensures that the correct gradient signal is propagated through the scaling layer to the individual pixels of the images.

Another idea we use to increase robustness and transferrability of the attacks is image augmentation. Denote by $T_{\theta}$ an image augmentation function with parameter $\theta$. For instance, we can have $\theta \in[0,2 \pi)$ as an angle and $T_{\theta}$ as the function that rotates the input image clock-wise by $\theta$. The parameter $\theta$ can also be a vector. For instance, we can have $\theta \in(0, \infty)^{2}$ as scaling factors in the width and height dimension, and $T_{\theta}$ as the function that scales the input image in the width direction by $\theta_{1}$ and in the height direction by $\theta_{2}$. In our final algorithm, $T_{\theta}$ takes the general form of a projective transformation with $\theta \in \mathbb{R}^{8}$ as implemented in tf.contrib.image.transform.

Let $x$ be an input image, and $y_{\text {true }}$ be the label of $x$. Our attack algorithm works to find an $x^{a d v}$ that maximizes the expected average cross entropy loss of the predictions of $f^{1}, \ldots, f^{k}$ over a random input augmentation 6

$$
\max _{x^{a d v}:\left\|x-x^{a d v}\right\|_{\infty} \leq \varepsilon} \mathbf{E}_{\theta}\left[\frac{1}{k} \sum_{i=1}^{k} J\left(f^{i}\left(T_{\theta}(x)\right), y_{\text {true }}\right)\right] .
$$

However, in a typical attack scenario, the true label $y_{\text {true }}$ is not available to the attacker, therefore we substitute it with a psuedo-label $\hat{y}$ generated by an image classifer $g$ that is available to the attacker. The objective of our attack is thus the following

$$
\max _{x^{a d v}:\left\|x-x^{a d v}\right\|_{\infty} \leq \varepsilon} \frac{1}{k} \sum_{i=1}^{k} \mathbf{E}_{\theta^{i}}\left[J\left(f^{i}\left(T_{\theta^{i}}(x)\right), g(x)\right)\right] .
$$

Using linearity of gradients, we write the gradient of the objective as

$$
\frac{1}{k} \sum_{i=1}^{k} \nabla_{x} \mathbf{E}_{\theta^{i}}\left[J\left(f^{i}\left(T_{\theta^{i}}(x)\right), g(x)\right)\right] .
$$

For typical distributions of $\theta$, such as uniform or normal distribution, the gradient of the expected cross entropy loss over a random $\theta$ is hard to compute. In our solution, we replace it with an empirical estimate which is an average of the gradients for a few samples of $\theta$. We also adopt the approach in [40] where $x$ is first randomly perturbed. The use of random projective transformation seems to be a natural idea, but to the best of our knowledge, this has not been explicitly described in previous works on generating adversarial examples for image classifiers.

In the rest of this section, we use $\widehat{\nabla}^{i}(x)$ to denote the empirical gradient estimate on input image $x$ as described above.

Let $x_{a d v}^{0}:=x, x^{\min }=\max (x-\varepsilon, 0), x^{\max }=\min (x+\varepsilon, 1)$, and let $\alpha^{1}, \alpha^{2}, \ldots$ be a sequence of pre-defined step sizes. Then in the $i$-th step of the iteration, we update the image by

\footnotetext{
${ }^{6}$ The distribution we use for $\theta$ corresponds to a small random augmentation. See code for details.
} 


$$
x_{a d v}^{i}=\operatorname{clip}\left(x_{a d v}^{i-1}+\alpha^{i} \operatorname{sign}\left(\frac{1}{k} \sum_{i=1}^{k} \widehat{\nabla}^{i}(x)\right), x^{\min }, x^{\max }\right) .
$$

Optimization We noticed from our experiments that non-targeted attacks against pre-trained networks without defense (white-box and black-box) typically succeed in $3-4$ rounds, whereas attacks against adversarially trained networks take more iterations. We also observed that in later iterations, there is little benefit in including in the ensemble un-defended networks that have been successfully attacked. In the final solution, each iteration is defined by step size $\alpha^{i}$ as well as the set of classifiers to include in the ensemble for the respective iteration. These parameters were found through trial and error on the official development dataset of the competition.

Experiments: non-targeted attack We randomly selected 18,000 images from ImageNet [32] for which Inception V3 [36] classified correctly.

The classifiers in the ensemble are: Inception V3 [36], ResNet 50 [17], ResNet 101 [17], Inception ResNet V2 [35], Xception [8], ensemble adversarially trained Inception ResNet V2 (EnsAdv Inception ResNet V2) [40], and adversarially trained Inception V3 (Adv Inception V3) [24].

We held out a few models to evaluate the transferrability of our attacks. The holdout models listed in Table 4 are: Inception V4 [35], ensemble adversarially trained Inception V3 with 2 (and 3) external models (Ens-3-Adv Inception V3, and Ens-4-Adv Inception V3, respectively) [40].

Table 4: Success rate - non-targeted attack

\begin{tabular}{lr}
\hline Classifier & Success rate \\
\hline Inception V3 & $96.74 \%$ \\
ResNet 50 & $92.78 \%$ \\
Inception ResNet V2 & $92.32 \%$ \\
EnsAdv Inception ResNet V2 & $87.36 \%$ \\
Adv Inception V3 & $83.73 \%$ \\
\hline Inception V4 & $91.69 \%$ \\
Ens-3-Adv Inception V3 & $62.76 \%$ \\
Ens-4-Adv Inception V3 & $58.11 \%$ \\
\hline
\end{tabular}

Table 4 lists the success rate for non-targeted attacks with $\varepsilon=16 / 255$. The performance for $\varepsilon=12 / 255$ is similar, and somewhat worse for smaller $\varepsilon$. We see that a decent amount of the generated attacks transfer to the two holdout adversarially trained network Ens-3-Adv Inception V3 and Ens-4-Adv Inception V3. The transfer rate for many other publicly available pretrained networks without defense are all close to or above $90 \%$. For brevity, we only list the performance on Inception V4 for comparison.

Targeted attack Our targeted attack follows a similar approach as non-targeted attack. The main differences are: 
1. For the objective, we now minimize the loss between a target label $y_{\text {target }}$, instead of maximizing with respect to $\hat{y}$ as in Equation (5.4).

2. Our experiments show that doing random image augmentation severely decreases the success rate for even white-box attacks, therefore no augmentation is performed for targeted-attacks. Note that here success is defined as successfully make the classifier output the target class. The attacks with image augmentation typically managed to cause the classifiers to output some wrong label other than the target class.

Our conclusion is that if the success criteria is to trick the classifier into outputting some specific target class, then our targeted attack does not transfer well and is not robust.

\subsection{3rd place in targeted attack track: team FatFingers}

Team members: Yao Zhao, Yuzhe Zhao, Zhonglin Han and Junjiajia Long

We propose a dynamic iterative ensemble targeted attack method, which builds iterative attacks on a loss ensemble neural networks focusing on the classifiers that are harder to perturb. Our methods are tested among 65 attackers against 107 defenders in NIPS-Kaggle competition and achieved 3rd in the targeted attack ranking.

\subsubsection{Targeted Attack Model Transfer}

In our experiments, we compared variants of single step attack methods and iterative attack methods including two basic forms of those two attack methods: fast gradient sign (FGS)

$$
\mathbf{x}^{a d v}=\mathbf{x}+\varepsilon \cdot \operatorname{sign}\left(\nabla_{x} J\left(f(\mathbf{x}), y_{\text {true }}\right)\right)
$$

and iterative sign attacks:

$$
\mathbf{x}_{t+1}^{a d v}=\operatorname{clip}_{\varepsilon, \mathbf{x}}\left\{\mathbf{x}_{t}^{a d v}+\alpha \cdot \operatorname{sign}\left(\nabla_{x} J\left(f\left(\mathbf{x}_{t}^{a d v}\right), y_{t r u e}\right)\right)\right\}
$$

To evaluate the ability of black-box targeted attacks, we built iterative attack methods (10 iterations) using single models against many single model defenders individually on 1000 images. Fig 4 demonstrates the matrix of target hitting for 10 attacking models, while Fig 5 shows their capabilitis of defending.

White-box targeted adversarial attacks are generally successful, even against adversarial trained models. Though targeted adversarial attacks built on single models lower the accuracy of defenders based on a different model, the hit rate are close to zero. 


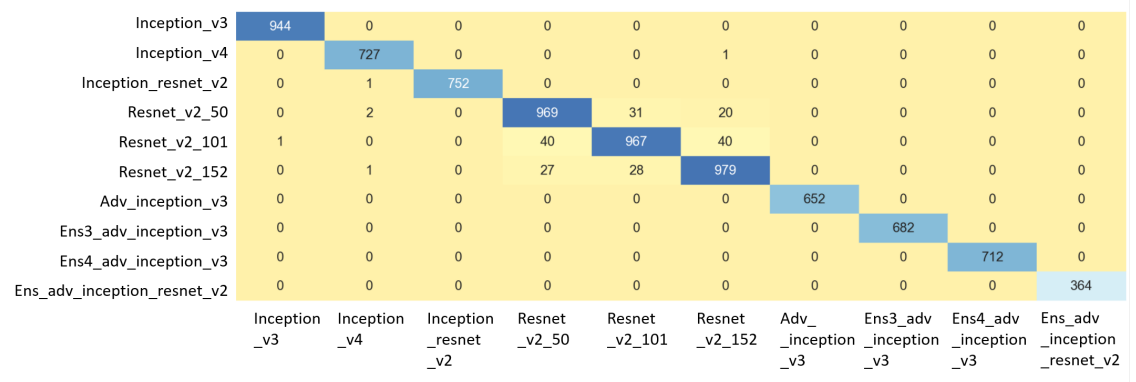

Fig. 4: Target Hitting Matrix

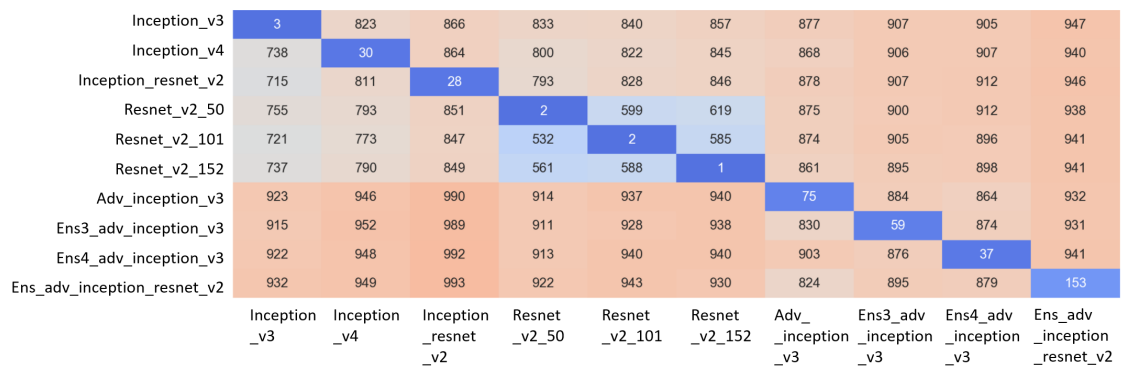

Fig. 5: Defender Accuracy Matrix

\subsubsection{Ensemble Attack Methods}

Since targeted attacks against unknown models has very low hit rate, it is important to combine known models in a larger number and more efficiently to attack a pool of unknown models or their ensembles.

Probability ensemble is a common way to combine a number of classifiers (sometimes called majority vote). However, the loss function is usually hard to optimize because the parameters of different classifiers are coupled inside the logarithm.

$$
J_{\text {prob }}(\mathbf{x}, y)=-\sum_{j}^{N} y_{j} \log \left(\frac{1}{M} \sum_{i}^{M} p_{i j}(\mathbf{x})\right)
$$


By Jensen's inequality, an upper bound is obtained for the loss function. Instead of minimizing $J_{\text {prob }}(\mathbf{x}, y)$, we propose to optimize the upper bound. This way of combining classifiers is called loss ensemble. By using the following new loss function eq.4, the parameters of different neural networks are decoupled, which helps the optimization.

$$
J_{\text {prob }}(\mathbf{x}, y) \leq-\frac{1}{M} \sum_{j}^{N} \sum_{i}^{M} y_{i j} \log \left(p_{i j}(\mathbf{x})\right)=J_{\text {loss }}(\mathbf{x}, y)
$$

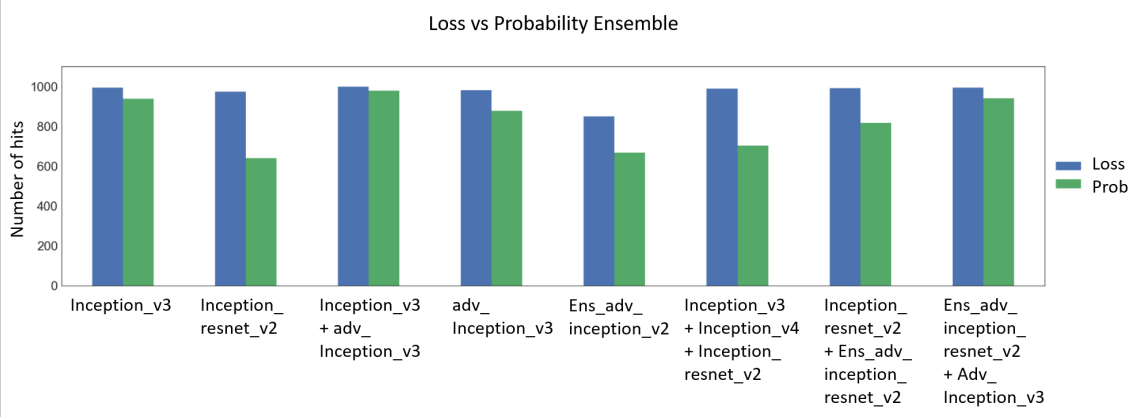

Fig. 6: Loss ensemble v.s. probability ensemble. Targeted attacks using the loss ensemble method outperforms probability ensemble at given number of iterations.

Comparisons between results of targeted attacks using loss ensemble and probability ensemble at given iterations were shown in Fig 6. In general, it demonstrates that capability of targeted attacking using loss ensemble is superior to that using probability ensemble.

\subsubsection{Dynamic Iterative Ensemble Attack}

The difficulty of attacking each individual neural network model within an ensemble can be quite different. We compared iterative attack methods with different parameters and found that number of iterations is most crucial, as shown in Fig.7. For example, attacking an adversarial trained model at high success rate takes significantly more iterations than normal models.

$$
\mathbf{x}_{t+1}^{a d v}=\operatorname{clip}_{\varepsilon, \mathbf{x}}\left\{\mathbf{x}_{t}^{a d v}+\alpha \cdot \operatorname{sign}\left(\frac{1}{M} \sum_{k}^{M} \delta_{t k} \nabla_{x} J_{k}\left(f\left(\mathbf{x}_{t}^{a d v}\right), y_{t r u e}\right)\right)\right\}
$$

For tasks where computation is limited, we implemented a method that preassigns the number of iterations for each model or dynamically adjusts whether 


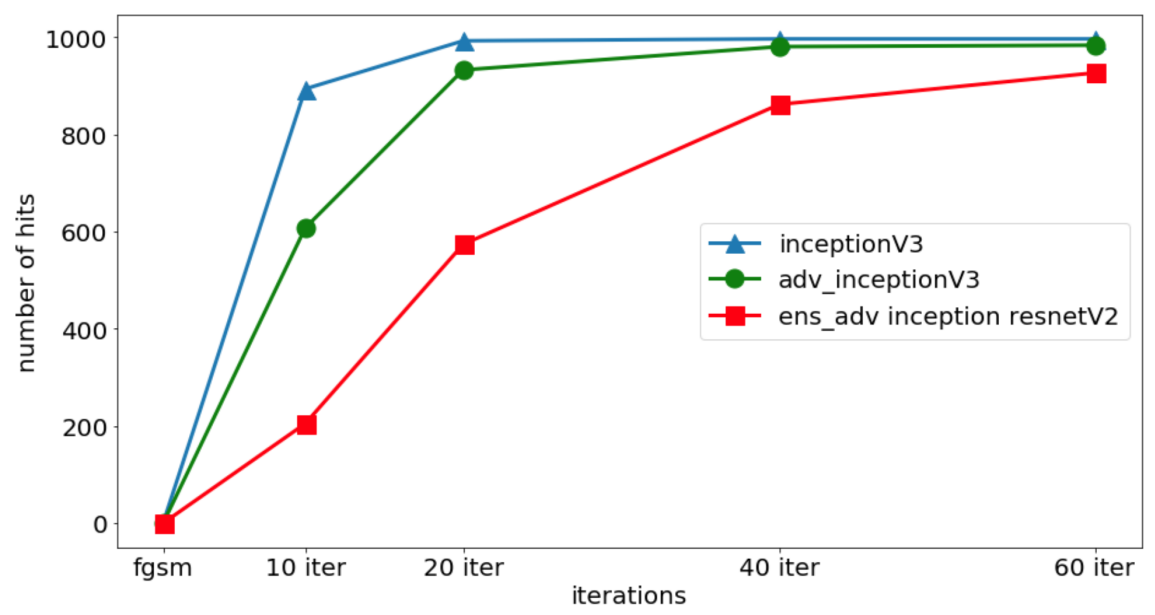

Fig. 7: Dynamic iterative ensemble attack results for three selected models

to include a model in each step of the attack by observing if the loss function for that model is small enough. As shown in Eq 22, $\delta_{t k} \in\{0,1\}$ determines if loss for model $k$ is included in the total loss at time step $t$.

\subsection{4th place in defense track: team erko}

Team members: Yerkebulan Berdibekov

In this section, I describe a very simple defense solution against adversarial attacks using spatial smoothing on the input of adversarially trained models. This solution took 4th place in the final round. Using spatially smoothing, in particularly median filtering with 2 by 2 windows on images and processing it by only adversarially trained models we can achieve simple and decent defense against black box attacks. Additionally this approach can work along with other defense solutions that use randomizations (data augmentations \& other types of defenses).

Adversarially trained models are models trained on adversarial examples along with a given original dataset. In the usual procedure for adversarial training, during the training phase half of each mini-batch of images are replaced with adversarial examples generated on the model itself (white box attacks). This can provide robustness against future white-box attacks. However, like described in [40] gradientmasking makes the finding of adversarial examples a challenging task. Due to this, adversarially trained models cannot guarantee robustness against black-box attacks. Many other techniques have been developed to overcome these problems. 


\subsubsection{Architecture of Defense Model}

Figure 8 below shows the architecture of my simple defense model: an input image is followed by median filtering, and then this filtered image is fed to ensemble of adversarially trained models. The resulting predictions are then averaged. However, like described in the sections below, many other variations of ensembles and single models were tested. The best results were achieved using an ensemble of all adversarially trained models with median filtering.

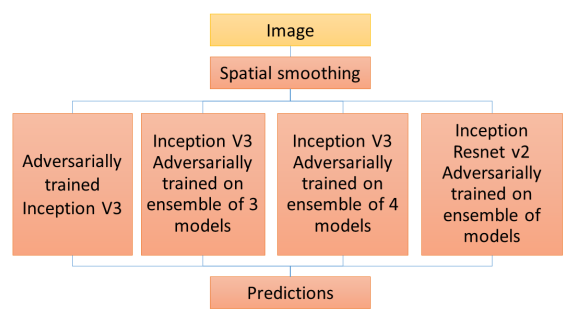

Fig. 8: Architecture of simple defense model, using median filtering with only adversarially trained models.

\subsubsection{Spatial smoothing: median filtering.}

Median filtering is often used in image/photo pre-processing to reduce noise while preserving edges and other features. It is robust against random high-magnitude perturbations resembling salt-and-pepper noise. Photographers also use median filtering to increase photo quality. ImageNet may contain many median filtered images. Other major advantages of image filtering include:

- Median filtering does not harm classification accuracy on clean examples, as shown below in experiments in Section 5.6.3

- Does not require additional expensive training procedures other than the adversarially trained model itself.

\subsubsection{Experiments}

I have experimentally observed that using median filtering only we cannot defend against strong adversarial attacks like described by Carlini and Wagner [?]. However, I have also experimentally observed that using median filtering and only adversarially trained models we can obtain a robust defense against adversarial attacks.

In my experiments I used the dataset provided by competition organizers and used a modified C\&W L2 attack to generate adversarial examples. These examples 
were later used to calculate the adversarial example misclassification ratio (number of wrong classifications divided by number of all examples) and to rank defenses. To generate adversarial examples I used either a single model or ensemble of models (a list of multiple models is indicated in each cell).

In all experiments I used a hold-out inception_v4 model that was not used to generate adversarial examples (see Table 5. Table 6. This allowed us to test transferability of attacks and to test spatial smoothing effects.

\subsubsection{Effects of median filtering}

On our holdout inception_v4 model, using median filtering performs nearly the same as without median filtering. Same results on other non-adversarially trained models. With median filtering or without, misclassification ratio differences are small.

Adversarially trained models with median filtering show good defense against attacks. An ensemble of these adversarially trained models with median filtered images is robust against black-box attacks and to attacks generated by an ensemble containing same models (see Table 5, Table 6). This is not exactly a white-box attack, because we generate adversarial examples on a model without a filtering layer. For example, we use a pre-trained ens3_adv_inception_v3 model to generate adversarial examples. These images are median filtered and fed to model again to check the misclassification ratios.

All these attacks were generated using $\varepsilon=16$ max pixel perturbations. In the case of the best ensemble defense against the best ensemble attacker, I tested other values of $\varepsilon$ and plotted Figure 9 showing that in case of lower $\varepsilon$ values this defense approach is more robust against attacks(exact values in Table 7):

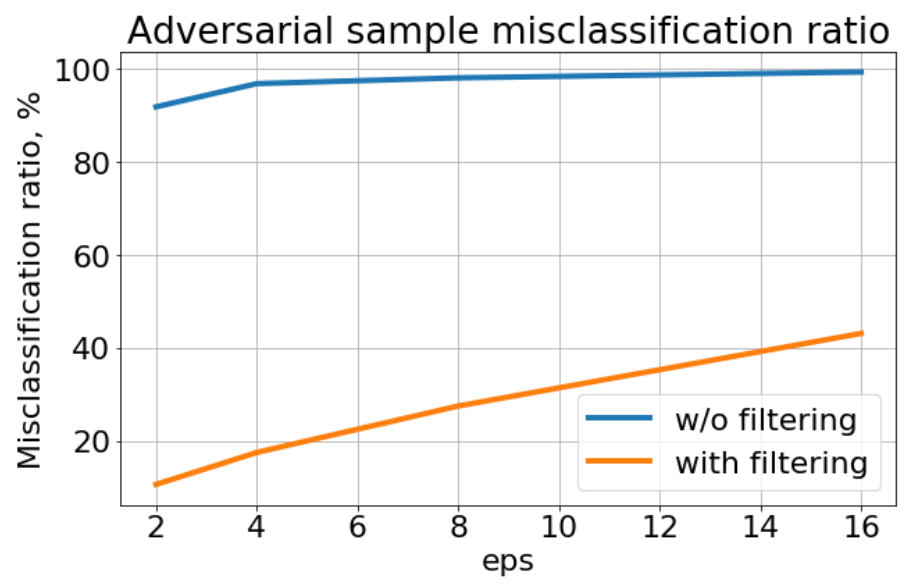

Fig. 9: Adversarial examples misclassification ratio, percentage 
Table 5: Misclassification ratio without filtering, percentage. Rows are defenders; columns are attackers. Even ensemble of adversarially trained models are not robust against good attackers.

\begin{tabular}{lcccc}
\hline Defenders $\backslash$ Attackers & inception_v3 & $A$ & $B$ & $C$ \\
\hline inception_v3 & 100.00 & 100.00 & 26.25 & 99.38 \\
\hline inception_v4 & 42.50 & 80.63 & 21.88 & 62.50 \\
\hline adv_inception_v3 & 20.62 & 41.25 & 100.00 & 100.00 \\
\hline ens3_adv_inception_v3 & 15.62 & 38.13 & 100.00 & 99.38 \\
\hline ens_adv_inception_resnet_v2 & 10.62 & 23.75 & 94.38 & 95.00 \\
\hline $\begin{array}{l}\text { adv_inception_v3 } \\
\text { ens3_adv_inception_v3 }\end{array}$ & 15.00 & 36.25 & 100.00 & 100.00 \\
\hline $\begin{array}{l}\text { adv_inception_v3 } \\
\text { ens3_adv_inception_v3 } \\
\text { ens4_adv_inception_v3 }\end{array}$ & 16.25 & 33.13 & 100.00 & 99.38 \\
\hline $\begin{array}{l}\text { adv_inception_v3 } \\
\text { ens3_adv_inception_v3 } \\
\text { ens_adv_inception_resnet_v2 } \\
\text { ens4_adv_inception_v3 }\end{array}$ & & & & \\
\hline
\end{tabular}

Where $A$ is an ensemble of inception_v3, inception_resnet_v2, resnet_v1_101, resnet_v1_50, resnet_v2_101, resnet_v2_50, vgg_16;

$B$ is an ensemble of adv_inception_v3, ens3_adv_inception_v3, ens_adv_inception_resnet_v2, ens4_adv_inception_v3;

$C$ is an ensember of inception_v3, adv_inception_v3, ens3_adv_inception_v3, ens_adv_inception_resnet_v2, ens4_adv_inception_v3, inception_resnet_v2, resnet_v1_101, resnet_v1_50, resnet_v2_101.

\subsubsection{Submission results}

Following the competition results, we have seen that adversarially trained models with median filtering are indeed robust to most types of attacks. These results suggest more study on this effect of adversarially trained models in the future.

During the competition, new types of attacks were developed with smoothed adversarial examples that can fool spatially smoothed defenses with as high as 50-60\% ratio and with high transferability. These are the best attackers developed in NonTargeted/Targeted Adversarial Attack Competitions. Additional study is needed to defend against these new types of attacks.

\subsection{4th place in non-targeted attack track: team iwiwi}

Team members: Takuya Akiba and Seiya Tokui and Motoki Abe

In this section, we explain the submission from team iwiwi to the non-targeted attack track. This team was Takuya Akiba, Seiya Tokui and Motoki Abe. The ap- 
Table 6: Misclassification ratio with filtering, percentage. Adversarially trained models with median filtering show better robustness against many kinds of attacks within these experiments. inception_v4 model with median filtering on all of attacks performs nearly same as without filtering. Same on other non-adversarial models. Therefore, I am speculating median filtering is not cleaning, or not mitigating adversarial examples.

\begin{tabular}{lcccc}
\hline Defenders $\backslash$ Attackers & inception_v3 & $A$ & $B$ & $C$ \\
\hline inception_v3 & 100.00 & 97.50 & 27.50 & 95.63 \\
\hline inception_v4 & 40.00 & 75.63 & 22.50 & 57.50 \\
\hline adv_inception_v3 & 21.88 & 43.13 & 33.13 & 40.00 \\
\hline ens3_adv_inception_v3 & 21.88 & 43.75 & 57.50 & 58.13 \\
\hline ens_adv_inception_resnet_v2 & 13.13 & 30.63 & 30.63 & 39.38 \\
\hline $\begin{array}{l}\text { adv_inception_v3 } \\
\text { ens3_adv_inception_v3 }\end{array}$ & 17.50 & 40.00 & 43.75 & 47.50 \\
\hline $\begin{array}{l}\text { adv_inception_v3 } \\
\text { ens3_adv_inception_v3 } \\
\text { ens4_adv_inception_v3 }\end{array}$ & 17.50 & 38.75 & 43.75 & 48.75 \\
\hline $\begin{array}{l}\text { adv_inception_v3 } \\
\text { ens3_adv_inception_v3 } \\
\text { ens_adv_inception_resnet_v2 } \\
\text { ens4_adv_inception_v3 }\end{array}$ & & & & \\
\hline
\end{tabular}

Where $A$ is an ensemble of inception_v3, inception_resnet_v2, resnet_v1_101, resnet_v1_50, resnet_v2_101, resnet_v2_50, vgg_16;

$B$ is an ensemble of adv_inception_v3, ens3_adv_inception_v3, ens_adv_inception_resnet_v2, ens4_adv_inception_v3;

$C$ is an ensemble of inception_v3, adv_inception_v3, ens3_adv_inception_v3, ens_adv_inception_resnet_v2, ens4_adv_inception_v3, inception_resnet_v2, resnet_v1_101, resnet_v1_50, resnet_v2_101.

Table 7: Misclassification ratio on $\varepsilon$ values, percentage. On smaller $\varepsilon$ values, median filtering shows even better robustness to adversarial attacks.

\begin{tabular}{lcccc}
\hline Defenders & $\varepsilon=16$ & $\varepsilon=8$ & $\varepsilon=4$ & $\varepsilon=2$ \\
\hline Ensemble of adversarial models non-filtered input & 99.375 & 98.125 & 96.875 & 91.875 \\
Ensemble of adversarial models with filtered input & 43.125 & 27.500 & 17.500 & 10.625 \\
\hline
\end{tabular}

proach is quite different from other teams: training fully-convolutional networks (FCNs) that can convert clean examples to adversarial examples. The team received the 4th place.

\subsubsection{Basic Framework}

Given a clean input image $x$, we generate an adversarial example as follows: 


$$
x^{a d v}=\operatorname{Clip}_{[0,1]}\left(x+a\left(x ; \theta_{a}\right)\right) .
$$

Here, $a$ is a differentiable function represented by a FCN with parameter $\theta_{a}$. We call $a$ as an attack $F C N$. It outputs $c \times h \times w$ tensors, where $c, h, w$ are the number of channels, height and width of $x$. The values of the output are in range $[-\varepsilon,+\varepsilon]$. During the training of the attack FCN, to confuse image classifiers, we maximize the loss $J\left(f\left(x^{a d v}\right), y\right)$, where $f$ is a pre-trained image classifier. We refer to $f$ as a target model. Specifically, we optimize $\theta_{a}$ to maximize the following value:

$$
\sum_{x \in \mathscr{X}} J\left(f\left(\operatorname{Clip}_{[0,1]}\left(x+a\left(x ; \theta_{a}\right)\right)\right), y\right) .
$$

This framework has some commonality with the work by Baluja and Fischer [1]. They also propose to train neural networks that produce adversarial examples. However, while we have the hard constraint on the distance between clean and adversarial examples, they considered the distance as one of optimization objective to minimize. In addition, we used a much larger FCN model and stronger computation power, together with several new ideas such as multi-target training, multi-task training, and gradient hints, which are explained in the next subsection.

\subsubsection{Empirical Enhancement}

Multi-Target Training. To obtain adversarial examples that generalize to different image classifiers, we use multiple target models to train the attack FCN. We maximize the sum of losses of all models. In this competition, we used eight models: (1) ResNet50, (2) VGG16, (3) Inception v3, (4) Inception v3 with adversarial training, (5) Inception v3 with ensemble adversarial training (EAT) using three models, (6) Inception v3 with EAT using four models, (7) Inception ResNet v2, and (8) Inception ResNet v2 with EAT. All of these classifier models are available online.

Multi-Task Training. A naive approach to construct a FCN so that it outputs values in the range $[-\varepsilon,+\varepsilon]$ is to apply the tanh function to the last output, and then multiply it by $\varepsilon$. However, in this way, the FCN cannot finely control the magnitude of perturbation, as $\varepsilon$ is not given to the FCN. To cope with this issue, we take the advantage of discreteness. In this competition, $\varepsilon$ can take 13 values: $\frac{4}{256}, \frac{5}{256}, \ldots, \frac{16}{256}$. We consider adversarial attack with different $\varepsilon$ values as different tasks, and employ multi-task training. Specifically, the FCN outputs a tensor with shape $13 \times c \times h \times w$, where the first dimension corresponds to the $\varepsilon$ value.

Gradient Hints. Attack methods that use the gradients on image pixels work well. Therefore, these gradients are useful signals for generating adversarial examples. Thus, in addition to clean examples, we also use these gradients as input to the FCN. In this competition, we used gradients by Inception ResNet v2 with EAT, which was the strongest defense model publicly available. 

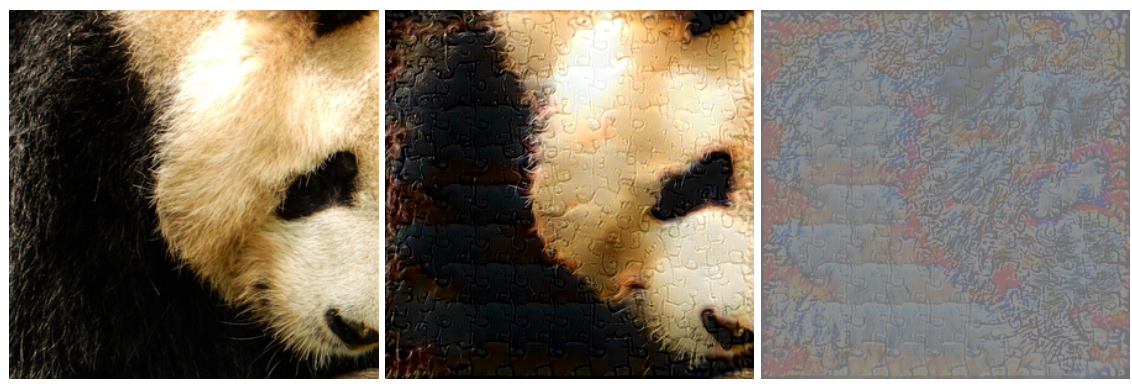

Fig. 10: A clean example (left), adversarial example generated by our method (middle), and their difference (right), where $\varepsilon=\frac{16}{255}$.

\subsubsection{Results and Discussion}

The team ranked 4th among about one hundred teams. In addition, the team ranked 1st in 3rd-party PageRank-like analysis 7 which shows that this attack method is especially effective for strong defense methods.

In addition to its effectiveness, the generated attack images have interesting appearance (Figure 10, more examples are available online ${ }^{8}$. We observe two properties from the generated images: detailed textures are canceled out, and Jigsawpuzzle-like patterns are added. These properties deceive image classifiers into answering the Jigsaw puzzle class.

\section{Conclusion}

Adversarial examples are interesting phenomenon and important problem in machine learning security. Main goals of this competition were to increase awareness of the problem and stimulate researchers to propose novel approaches.

Competition definitely helped to increase awareness of the problem. Article "AI Fight Club Could Help Save Us from a Future of Super-Smart Cyberattacks" 9 was published in MIT Technology review about the competition. And more than 100 teams were competing in the final round.

Competition also pushed people to explore new approaches and improve existing methods to the problem. In all three tracks, competitors showed significant improvements on top of provided baselines by the end of the competition. Additionally, top submission in the defense tracked showed 95\% accuracy on all adversarial images produced by all attacks. While worst case accuracy was not as good as an average

$\overline{7}$ https://www.kaggle.com/anlthms/pagerank-ish-scoring

8 https://github.com/pfnet-research/nips17-adversarial-attack

9 www.technologyreview.com/s/608288 
accuracy, the results are still suggesting that practical applications may be able to achieve reasonable level of robustness to adversarial examples in black box case.

\section{References}

1. S. Baluja and I. Fischer. Adversarial transformation networks: Learning to generate adversarial examples. 2017.

2. B. Biggio, I. Corona, D. Maiorca, B. Nelson, N. Šrndić, P. Laskov, G. Giacinto, and F. Roli. Evasion attacks against machine learning at test time. In Joint European Conference on Machine Learning and Knowledge Discovery in Databases, pages 387-402. Springer, 2013.

3. W. Brendel, J. Rauber, and M. Bethge. Decision-based adversarial attacks: Reliable attacks against black-box machine learning models. 2017.

4. J. Buckman, A. Roy, C. Raffel, and I. Goodfellow. Thermometer encoding: One hot way to resist adversarial examples. Submissions to International Conference on Learning Representations, 2018.

5. N. Carlini and D. Wagner. Adversarial examples are not easily detected: Bypassing ten detection methods. In USENIX Workshop on Offensive Technologies, 2017.

6. N. Carlini and D. Wagner. Towards evaluating the robustness of neural networks. IEEE Symposium on Security and Privacy, 2017.

7. P.-Y. Chen, H. Zhang, Y. Sharma, J. Yi, and C.-J. Hsieh. Zoo: Zeroth order optimization based black-box attacks to deep neural networks without training substitute models. 2017.

8. F. Chollet. Xception: Deep learning with depthwise separable convolutions, 2016.

9. N. Das, M. Shanbhogue, S.-T. Chen, F. Hohman, L. Chen, M. E. Kounavis, and D. H. Chau. Keeping the bad guys out: Protecting and vaccinating deep learning with jpeg compression. arXiv preprint arXiv:1705.02900, 2017.

10. J. Deng, W. Dong, R. Socher, L.-J. Li, K. Li, and L. Fei-Fei. Imagenet: A large-scale hierarchical image database. In Computer Vision and Pattern Recognition, 2009. CVPR 2009. IEEE Conference on, pages 248-255. IEEE, 2009.

11. Y. Dong, F. Liao, T. Pang, H. Su, X. Hu, J. Li, and J. Zhu. Boosting adversarial attacks with momentum. arXiv preprint arXiv:1710.06081, 2017.

12. W. Duch and J. Korczak. Optimization and global minimization methods suitable for neural networks. Neural computing surveys, 2:163-212, 1998.

13. N. F. Geoffrey E Hinton, Sara Sabour. Matrix capsules with em routing. In International Conference on Learning Representations, 2018.

14. J. Gilmer, L. Metz, F. Faghri, S. S. Schoenholz, M. Raghu, M. Wattenberg, and I. Goodfellow. Adversarial spheres. Submissions to International Conference on Learning Representations, 2018.

15. I. J. Goodfellow, J. Shlens, and C. Szegedy. Explaining and harnessing adversarial examples. CoRR, abs/1412.6572, 2014.

16. I. J. Goodfellow, J. Shlens, and C. Szegedy. Explaining and harnessing adversarial examples. CoRR, abs/1412.6572, 2014.

17. K. He, X. Zhang, S. Ren, and J. Sun. Deep residual learning for image recognition, 2015.

18. K. He, X. Zhang, S. Ren, and J. Sun. Identity mappings in deep residual networks. In ECCV, 2016.

19. W. He, J. Wei, X. Chen, N. Carlini, and D. Song. Adversarial example defense: Ensembles of weak defenses are not strong. In 11th USENIX Workshop on Offensive Technologies (WOOT 17), Vancouver, BC, 2017. USENIX Association.

20. R. Huang, B. Xu, D. Schuurmans, and C. Szepesvári. Learning with a strong adversary. CoRR, abs/1511.03034, 2015.

21. D. Kingma and J. Ba. Adam: A method for stochastic optimization. arXiv preprint arXiv:1412.6980, 2014. 
22. A. Kurakin, I. Goodfellow, and S. Bengio. Adversarial examples in the physical world. In ICLR'2017 Workshop, 2016.

23. A. Kurakin, I. Goodfellow, and S. Bengio. Adversarial examples in the physical world. In ICLR'2017 Workshop, 2016.

24. A. Kurakin, I. J. Goodfellow, and S. Bengio. Adversarial machine learning at scale. In ICLR'2017, 2016.

25. F. Liao, M. Liang, Y. Dong, T. Pang, J. Zhu, and X. Hu. Defense against adversarial attacks using high-level representation guided denoiser. arXiv preprint arXiv:1712.02976, 2017.

26. Y. Liu, X. Chen, C. Liu, and D. Song. Delving into transferable adversarial examples and black-box attacks. In Proceedings of 5th International Conference on Learning Representations, 2017.

27. A. Madry, A. Makelov, L. Schmidt, D. Tsipras, and A. Vladu. Towards deep learning models resistant to adversarial attacks. 2017.

28. J. H. Metzen, T. Genewein, V. Fischer, and B. Bischoff. On detecting adversarial perturbations. In ICLR, 2017.

29. N. Papernot, P. McDaniel, and I. Goodfellow. Transferability in Machine Learning: from Phenomena to Black-Box Attacks using Adversarial Samples. ArXiv e-prints, May 2016b.

30. N. Papernot, P. McDaniel, I. Goodfellow, S. Jha, Z. B. Celik, and A. Swami. Practical blackbox attacks against machine learning. In Proceedings of the 2017 ACM on Asia Conference on Computer and Communications Security, ASIA CCS '17, pages 506-519, New York, NY, USA, 2017. ACM

31. B. T. Polyak. Some methods of speeding up the convergence of iteration methods. USSR Computational Mathematics and Mathematical Physics, 4(5):1-17, 1964.

32. O. Russakovsky, J. Deng, H. Su, J. Krause, S. Satheesh, S. Ma, Z. Huang, A. Karpathy, A. Khosla, M. Bernstein, A. C. Berg, and L. Fei-Fei. Imagenet large scale visual recognition challenge. International Journal of Computer Vision, 115(3):211-252, Dec 2015.

33. M. Sharif, S. Bhagavatula, L. Bauer, and M. K. Reiter. Accessorize to a crime: Real and stealthy attacks on state-of-the-art face recognition. In Proceedings of the 23rd ACM SIGSAC Conference on Computer and Communications Security, Oct. 2016. To appear.

34. I. Sutskever, J. Martens, G. Dahl, and G. Hinton. On the importance of initialization and momentum in deep learning. In ICML, 2013.

35. C. Szegedy, S. Ioffe, V. Vanhoucke, and A. A. Alemi. Inception-v4, inception-resnet and the impact of residual connections on learning. In $A A A I, 2017$.

36. C. Szegedy, V. Vanhoucke, S. Ioffe, J. Shlens, and Z. Wojna. Rethinking the inception architecture for computer vision, 2015.

37. C. Szegedy, V. Vanhoucke, S. Ioffe, J. Shlens, and Z. Wojna. Rethinking the inception architecture for computer vision. In CVPR, 2016.

38. C. Szegedy, W. Zaremba, I. Sutskever, J. Bruna, D. Erhan, I. Goodfellow, and R. Fergus. Intriguing properties of neural networks. In International Conference on Learning Representations, 2014.

39. C. Szegedy, W. Zaremba, I. Sutskever, J. Bruna, D. Erhan, I. J. Goodfellow, and R. Fergus. Intriguing properties of neural networks. ICLR, abs/1312.6199, 2014.

40. F. Tramr, A. Kurakin, N. Papernot, I. Goodfellow, D. Boneh, and P. McDaniel. Ensemble adversarial training: Attacks and defenses. In arxiv, 2017.

41. P. Vincent, H. Larochelle, Y. Bengio, and P.-A. Manzagol. Extracting and composing robust features with denoising autoencoders. In International Conference on Machine learning, pages 1096-1103, 2008.

42. C. Xie, J. Wang, Z. Zhang, Z. Ren, and A. Yuille. Mitigating adversarial effects through randomization. In International Conference on Learning Representations, 2018.

43. W. Xu, D. Evans, and Y. Qi. Feature squeezing: Detecting adversarial examples in deep neural networks. CoRR, abs/1704.01155, 2017.

44. K. Zhang, W. Zuo, Y. Chen, D. Meng, and L. Zhang. Beyond a gaussian denoiser: Residual learning of deep cnn for image denoising. IEEE Transactions on Image Processing, 2017. 